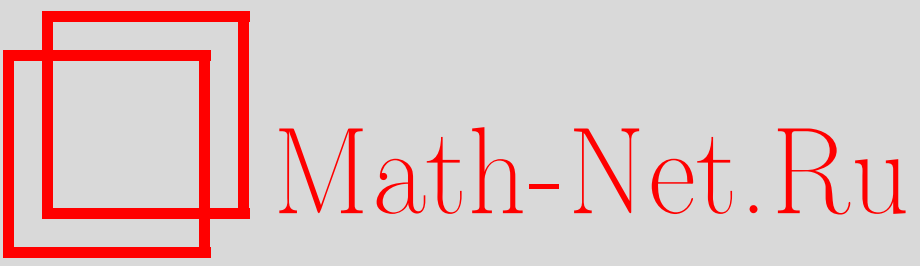

Э. Ю. Лернер, М. Д. Миссаров, Адельные фейнмановские амплитуды в низших порядках теории возмущений, ТМФ, 2000, том 124, номер 1, 95-109

DOI: https://doi.org/10.4213/tmf628

Использование Общероссийского математического портала Math-Net.Ru подразумевает, что вы прочитали и согласны с пользовательским соглашением

http://www.mathnet.ru/rus/agreement

Параметры загрузки:

IP: 54.81 .137 .203

26 апреля 2023 г., 18:11:14 
ТЕОРЕТИЧЕСКАЯ

И МАТЕМАТИЧЕСКАЯ

ФИЗИКА

Том 124, № 1

июль, 2000

(C) 2000 г.

Э.ю. Лернер*, М. Д. Миссаров*

\section{АДЕЛЬНЫЕ ФЕЙНМАНОВСКИЕ АМПЛИТУДЫ В НИЗШИХ ПОРЯДКАХ ТЕОРИИ ВОЗМУШЕНИЙ}

Сформулированы условия сходимости адельных фейнмановских амплитуд и доказано, что при достаточно больших значениях размерности пространства существует непустая область в пространстве степеней пропагаторов, в которой адельная амплитуда корректно определена. Исследуется аналитическое продолжение по степени пропагатора некоторых амплитуд $\varphi^{4}$-теории в третьем и четвертом порядках теории возмущений. Показано, что эти амплитуды не могут быть продолжены во всю комплексную плоскость (при условии справедливости гипотезы Римана о нулях дзета-функции), а интересные с физической точки зрения значения степени пропагатора могут лежать на границе области аналитичности.

\section{1. ВВЕДЕНИЕ}

В последние десять лет в математической физике стали рассматриваться различные $p$-адические и адельные аналоги моделей теории поля и статистической физики [1-4]. Ультраметричность $p$-адического расстояния приводит к замечательным упрощениям, что позволяет иногда давать более глубокое и детальное описание соответствующих моделей. Существует гипотеза о том, что евклидовы и $p$-адические модели поля могут синтезироваться в модели на группе аделей $[5,6]$. Строгий анализ адельной формулы Фройнда-Виттена для четырехточечной амплитуды Венециано был проведен в работах Владимирова [7]. К адельной гипотезе подводит также тот факт, что формальное описание скейлинг-инвариантных полей в евклидовом пространстве схоже с описанием полей в $p$-адическом пространстве [2]. Поэтому интересно попытаться изучить (хотя бы в первых порядках теории возмушений) фейнмановские амплитуды на группе аделей.

Напомним некоторые определения и результаты статьи [8], которые мы будем использовать.

Пусть $\mathbb{R}^{d}-d$-мерное вещественное пространство с обычной евклидовой нормой $\|\cdot\|_{\infty}$, а $Q_{p}^{d}-d$-мерное $p$-адическое пространство с нормой

$$
\left\|x_{p}\right\|_{p}=\max _{i=1, \ldots, d}\left\|x_{p}^{i}\right\|_{p}, \quad x_{p}=\left(x_{p}^{(1)}, \ldots, x_{p}^{(d)}\right) \in Q_{p}^{d}, \quad p=2,3, \ldots,
$$

\footnotetext{
* Казанский государственный университет, Казань, Россия. E-mail: Moukadas.Missarov@ksu.ru
} 
где $\|\cdot\|_{p}$ в правой части равенства обозначает $p$-адическую норму на $Q_{p}$, компоненты вектора $x_{p}$ являются $p$-адическими числами. Пусть $Z_{p}^{d}=\left\{x_{p} \in Q_{p}^{d}:\left\|x_{p}\right\|_{p} \leqslant 1\right\}$.

Будем назьвать $d$-мерной группой аделей $A^{d}$ ограниченное прямое произведение аддитивных групп $\mathbb{R}^{d}, Q_{p}^{d}, \quad p=2,3,5, \ldots$, по отношению к аддитивным группам $Z_{p}^{d}$, $p=2,3,5, \ldots$. Другими словами, $A^{d}$ есть множество всех последовательностей вида $x=\left(x_{\infty}, x_{2}, x_{3}, \ldots, x_{p}, \ldots\right)$, где $x_{\infty} \in \mathbb{R}^{d}, x_{p} \in Q_{p}^{d}, \quad p=2,3,5, \ldots$, и $x_{p} \in Z_{p}^{d}$ для всех $p$, кроме конечного их числа. Множество таких последовательностей образует группу относительно покомпонентного сложения. Последовательность аделей $x^{(n)}=$ $\left(x_{\infty}^{(n)}, x_{2}^{(n)}, \ldots, x_{p}^{(n)}, \ldots\right)$ сходится к аделю $x=\left(x_{\infty}, x_{2}, \ldots, x_{p}, \ldots\right)$, если она сходится к $x$ покомпонентно и сушествует такое $N$, что $x_{p}-x_{p}^{(n)} \in Z_{p}^{d}$ при $n \geqslant N$. Полученная топологическая группа $A^{d}$ локально-компактна.

Одномерную группу аделей $A^{1}$ называют еше кольцом аделей, поскольку в этом случае сушествует операция покомпонентного умножения аделей. Аддитивную группу этого кольца называют группой аделей.

Группа $A^{d}$ обладает инвариантной мерой, которую мы обозначим $d x$. Нормируем эту меру условием теs $\Omega^{d}=1$, где

$$
\Omega^{d}=\left\{x \in A^{d}: 0 \leqslant x_{\infty}^{1}<1, \ldots, 0 \leqslant x_{\infty}^{d}<1,\left\|x_{p}\right\|_{p} \leqslant 1, p=2,3,5, \ldots\right\} .
$$

Пространство Шварца $S\left(\mathbb{R}^{d}\right)$ задает пространство основных функций на $\mathbb{R}^{d}$. Пространства $D\left(Q_{p}^{d}\right)$ (пространства комплекснозначных локально-постоянных функций с соответствуюшей топологией сходимости $[3,9]), p=2,3, \ldots$, являются пространствами основных функций на $Q_{p}^{d}$.

Напомним определение пространства основных функций на группе аделей $A^{d}[9]$. Назовем элементарной функцией $h(x), x \in A^{d}$, функцию, представимую в виде бесконечного произведения

$$
h(x)=h_{\infty}\left(x_{\infty}\right) h_{2}\left(x_{2}\right) \ldots h_{p}\left(x_{p}\right) \ldots,
$$

где $h_{\infty} \in S\left(\mathbb{R}^{d}\right), h_{p}\left(x_{p}\right) \in D\left(Q_{p}^{d}\right), p=2,3,5, \ldots$, и $h_{p}\left(x_{p}\right)=\chi_{p}\left(x_{p}\right)$ для всех $p$, кроме конечного их числа. Здесь $\chi_{p}\left(x_{p}\right)$ - характеристическая функция шара $Z_{p}^{d}$. Пространство основных функций $S\left(A^{d}\right)$ на $A^{d}$, называемое пространством Шварца-Брюа, определяется как пространство всех конечных линейных комбинаций элементарных функций. Последовательность основных функций $h_{n}(x) \rightarrow 0$ при $n \rightarrow \infty$, если сушествует такое представление

$$
h_{n}(x)=\sum_{k=1}^{m} h_{k, \infty}^{(n)} h_{k, 2}^{(n)} \ldots h_{k, p}^{n} \ldots
$$

где $m$ не зависит от $n$, и сушествует такое $N$, что $h_{k, p}^{(n)}=\chi_{p}$ для всех $p>N$ и всех $n$ и $h_{k, \infty}^{(n)} \rightarrow 0$ в топологии $S\left(\mathbb{R}^{d}\right), h_{k, p}^{(n)} \rightarrow 0$ в топологии $D\left(Q_{p}^{d}\right)$ для $p \leqslant N$.

Определим модуль аделя $x \in A^{d}$ как

$$
|x|=\left\|x_{\infty}\right\|_{\infty} \prod_{p=2,3, \ldots}\left\|x_{p}\right\|_{p}
$$


Как легко следует из леммы Бореля-Кантелли, модуль $|x|$ определен для почти всех $x \in A^{d}$ при $d>1[8]$.

Чтобы избежать трудностей, связанных с определением модуля в одномерном случае, в работах $[10,11]$ было введено семейство обобщенных функций на пространстве Шварца-Брюа $S\left(A^{d}\right)$

$$
g(x ; \alpha, d)=c(\alpha ; d)|x|^{\alpha},
$$

где $\alpha \in \mathbb{C}_{\text {и }}$

$$
\begin{aligned}
c(\alpha ; d) & =c_{\infty}(\alpha ; d) \prod_{p} c_{p}(\alpha ; d), \\
\left(c_{p}(\alpha ; d)\right)^{-1} & =\int_{\left\|x_{p}\right\|_{p} \leqslant 1} d x_{p}=\frac{1-p^{-d}}{1-p^{-(\alpha+d)}}, \\
c_{\infty}(\alpha ; d) & =\frac{\pi^{-d / 2} \Gamma(d / 2)}{\pi^{(\alpha+d) / 2} \Gamma((\alpha+d) / 2)},
\end{aligned}
$$

где $Г(\alpha)$ - гамма-функция Эйлера. Заметим, что

$c(\alpha ; d)=\frac{\pi^{-d / 2} \Gamma(d / 2) \prod_{p}\left(1-p^{-d}\right)^{-1}}{\pi^{-(\alpha+d) / 2} \Gamma((\alpha+d) / 2) \prod_{p}\left(1-p^{-(\alpha+d)}\right)^{-1}}=\frac{\pi^{d / 2} \Gamma(d / 2) \zeta(d)}{\pi^{(\alpha+d) / 2} \Gamma((\alpha+d) / 2) \zeta(\alpha+d)}$,

где $\zeta(d)$ - дзета-функция Римана

В дальнейшем мы будем пользоваться обозначениями

$$
\begin{gathered}
\zeta_{p}(s)=\frac{1}{1-p^{-s}}, \quad p=2,3,5, \ldots, \\
\zeta_{\infty}(s)=\pi^{-\frac{s}{2}} \Gamma\left(\frac{s}{2}\right), \\
\Lambda(s)=\zeta_{\infty}(s) \prod_{p} \zeta_{p}(s)
\end{gathered}
$$

(в статье [8] использовались другие обозначения). Символ $\prod_{p} \zeta_{p}$ обозначает бесконечное произведение $\zeta_{p}$ по всем простым $p$, символ $\prod_{p=2}^{\infty} \zeta_{p}$-произведение

$$
\prod_{p=2}^{\infty} \zeta_{p}=\zeta_{\infty} \prod_{p} \zeta_{p}
$$

Действие обобшенной функции $g(a ; \alpha, d)$ на элементарную функцию $h=\prod_{p=2}^{\infty} h_{p}$ определим как

$$
(g(a ; \alpha, d), h(a))=\prod_{p=2}^{\infty} \frac{\zeta_{p}(d)}{\zeta_{p}(\alpha+d)} \int_{Q_{p}^{d}}\left\|x_{p}\right\|_{p}^{\alpha} h_{p}\left(x_{p}\right) d x_{p}
$$

4 Теоретическая и математическая физика, т. 124, № 1, 2000 г. 
где $Q_{\infty}^{d} \equiv \mathbb{R}^{d}$. Начиная с некоторого $p$

$$
h_{p}\left(x_{p}\right)=\chi_{p}\left(x_{p}\right), \quad \int_{\left\|x_{p}\right\| \leqslant 1}\left\|x_{p}\right\|_{p}^{\alpha} d x_{p}=\frac{\zeta_{p}(d)}{\zeta_{p}(\alpha+d)},
$$

и поэтому в бесконечном произведении (2) почти все сомножители равны единице.

Заметим, что все компоненты бесконечного произведения (2) являются целыми функциями от $\alpha$ при любых $d$, и, таким образом, обобшенная функция $g(\cdot ; \alpha, d)$ является целой функцией от $\alpha$.

Можно показать $[10,11]$, что при $2 d>\alpha>d$ гауссовское случайное поле с бинарной корреляционной функцией

$$
\langle\varphi(x) \varphi(y)\rangle=g(x-y ; \alpha-2 d, d)
$$

является трансляционно-инвариантным и скейлинг-инвариантным (с параметром $\alpha$ ) обобщенным случайным полем на группе аделей $A^{d}$. Скейлинг-преобразование $S_{\lambda}(\alpha)$ с параметром $\alpha$ определяется формулой

$$
S_{\lambda}(\alpha) \varphi(x)=|\lambda|^{d-\alpha / 2} \varphi(\lambda x)
$$

где $\lambda$ принадлежит группе аделей (обратимых элементов кольца $A^{1}$ ),

$$
\lambda x \equiv\left(\lambda_{\infty} x_{\infty}, \lambda_{2} x_{2}, \ldots\right), \quad \lambda_{p} x_{p}=\left(\lambda_{p} x_{p}^{1}, \ldots, \lambda_{p} x_{p}^{d}\right)
$$

В гиббсовской форме гауссовское поле с бинарной корреляционной функцией (3) задается гамильтонианом

$$
H_{0}(\varphi)=\frac{1}{2} \int \frac{\Lambda(2 d-\alpha) \Lambda(\alpha)}{\Lambda^{2}(d)} g(x-y ;-\alpha, d) \varphi(x) \varphi(y) d x d y
$$

По аналогии с евклидовой и $p$-адическими моделями естественно искать негауссовские скейлинг-инвариантные поля как возмушение гауссовского поля $\varphi^{4}$-взаимодействием:

$$
H(\varphi)=H_{0}(\varphi)+\int \varphi^{4}(x) d x
$$

Первым шагом в реализации этой программы является исследование фейнмановских диаграмм $\varphi^{4}$-теории.

В случае, если $\alpha=2+d, d=4-\varepsilon$, диаграммы теории с гамильтонианом (4) вплоть до третьего порядка теории возмушений изучались в работе [8] (см. также [11]). Была изучена структура полюсов этих диаграмм и доказана их перенормируемость. Исследование аналитических свойств адельных амплитуд сводится к исследованию бесконечного произведения евклидовой и всех $p$-адических фейнмановских амплитуд. При этом было обнаружено замечательное свойство, состоящее в том, что все полюсы евклидовой амплитуды, кроме основного полюса $\varepsilon=0$, являются нулями (того же порядка) 
бесконечного произведения $p$-адических амплитуд, и поэтому в адельной амплитуде эти значения не являются полюсами.

В разделе 1 мы исследуем произвольные адельные фейнмановские амплитуды как обобщенные функции на пространстве Шварца-Брюа и формулируем условия сходимости соответствующих фейнмановских интегралов. Показано, что при достаточно больших $d$ существует непустая область в пространстве степеней пропагаторов, в которой адельная амплитуда корректно определена. Для амплитуд $\varphi^{4}$-теории область сходимости непуста и в случае равных степеней пропагаторов при условии, что соответствуюшая диаграмма не содержит двухвосток. В разделе 2 мы исследуем аналитическое продолжение по степени пропагатора некоторых амплитуд $\varphi^{4}$-теории в третьем и четвертом порядках теории возмушений. Показано, что эти амплитуды не могут быть продолжены во всю комплексную плоскость (при условии справедливости гипотезы Римана о нулях дзета-функции). Более того, интересные с физической точки зрения значения степени пропагатора могут лежать на границе области аналитичности.

\section{2. СХОДИМОСТЬ ФЕЙНМАНОВСКИХ АДЕЛЬНЫХ АМПЛИТУД}

В дальнейшем, чтобы избежать громоздких нормируюших множителей в гауссовском корреляторе, мы будем предполагать, что $d>1$, и рассматривать фейнмановские амплитуды $\varphi^{4}$-теории с пропагатором $|x-y|^{\alpha-2 d}$.

Пусть $G=(V, L)$ - связный фейнмановский граф, $L(G)$ - множество ребер, $V(G)$ множество вершин графа. Последнее делится на две части: множество внутренних вершин $V_{\text {int }}$ и множество внешних вершин графа $V_{\text {ext }}$. Обозначим через $i(l)$ и $f(l)$ начало и конец ребра $l, l \in L(G)$. Подграф $H$ называется регулярным, если он содержит все ребра $l$ исходного граф̆а такие, что $i(l)$ и $f(l)$ принадлежат $V(H)$. Подграф̆ $H$ графа $G$ называется остовным, если $V(H)=V(G)$. Пусть $H$ - остовной подграф графа $G$, каждая компонента связности которого представляет собой регулярный подграф. Будем употреблять обозначение $G / H$ для фактор-графа графа $G$ по подграфу $H$. Обозначим через $\widehat{G / H}$ граф, получаюшийся из графа $G / H$ отождествлением всех его внешних вершин.

Для того чтобы определить адельную фейнмановскую амплитуду как обобщенную функцию на пространстве Шварца-Брюа, рассмотрим обобшенную адельную амплитуду вида

$$
F_{G}(\underline{\alpha})\left(x_{v}, v \in V_{\mathrm{ext}}\right)=\int_{\left(A^{d}\right)} V_{\mathrm{int}} \prod_{l \in L(G)}\left|x_{i(l)}-x_{f(l)}\right|^{\alpha_{l}-2 d} \prod_{v \in V_{\mathrm{int}}} d x_{v}
$$

где $\underline{\alpha}=\left(\alpha_{l}, l \in L(G)\right)$ - вектор комплексных параметров. Действие адельной амплитуды $F_{G}(\underline{\alpha})$ на основную функцию $h$ из пространства Шварца-Брюа на $\left(A^{d}\right)^{V_{\text {ext }}}$ задается формулой

$$
\left(F_{G}(\underline{\alpha}), h\right)=\int_{\left(A^{d}\right)^{V_{\mathrm{ext}}}} F_{G}(\underline{\alpha})\left(x_{v}, v \in V_{\mathrm{ext}}\right) h\left(x_{v}, v \in V_{\mathrm{ext}}\right) \prod_{v \in V_{\mathrm{ext}}} d x_{v} .
$$


Пусть

$$
f_{G, p}(\underline{\alpha})=\int_{\left(Q_{p}^{d}\right)^{V}} \prod_{l \in L(G)}\left\|x_{i(l), p}-x_{f(l), p}\right\|^{\alpha_{l}-2 d} \prod_{v \in V_{\mathrm{int}}} d x_{v, p} \prod_{v \in V_{\mathrm{ext}}} \chi_{p}\left(x_{v, p}\right) d x_{v, p},
$$

где $p=2,3, \ldots, \infty$ (при $p=\infty$ под функцией $\chi_{p}\left(x_{p}\right)$ будем понимать плотность стандартного гауссовского распределения). Исследование вопроса о сходимости интеграла $\left(F_{G}(\underline{\alpha}), h\right)$ сводится к вопросу о сходимости интегралов $f_{G, p}(\underline{\alpha})$ для $p=2,3, \ldots, \infty$ и бесконечного произведения

$$
f_{G}(\underline{\alpha})=\prod_{p} f_{G, p}(\underline{\alpha}) .
$$

Индекс связного графа $G[12,13]$ выражается через переменные $\alpha_{l}$ формулой

$$
\operatorname{Ind}(G)=-\sum_{l \in L(G)} \alpha_{l}+d(2|L(G)|-|V(G)|+1)
$$

Напомним, что нетривиальный связный подграф $H$ графа $G$ называется отделимым, если любой путь из вершины $v \in V(H)$ в вершину $v^{\prime} \in V(G) \backslash V(H)$ проходит через фиксированную вершину $v_{0} \in V(H)$, причем все вершины подграфа $H$, за исключением, может быть, вершины $v_{0}$, являются внутренними вершинами графа $G$.

Теорема 1. Интеграл (5) сходится, если

$$
\operatorname{Re} \operatorname{Ind}(H)<-1
$$

для всех нетривиальных регулярных связных подграфов $H$ графа $G$ и

$$
\operatorname{Re} \operatorname{Ind}(\widehat{G / H})>1
$$

для всех собственных подграфов $H$ графа $G$ таких, что каждая компонента связности графа $H$ является регулярным подграфом графа $G$, и граф $\widehat{G / H}$ не содержит петель. Для любого графа $G$, не содержащего отделимьх подграфов, при достаточно большом d существует непустая область в пространстве параметров $\underline{\alpha}$, для которой интеграл (5) сходится.

ДОКАЗАТЕЛЬСТво. Из неравенств (6), (7) следует положительность ультрафиолетовых и отрицательность инфракрасных индексов подграфов, что, как известно (см. [12-14]), является достаточным условием сходимости интегралов $f_{G, p}$ в вешественном и $p$-адических случаях. Для $p$-адического интеграла $f_{G, p}(\underline{\alpha})$ справедлива формула [15]

$$
f_{G, p}(\underline{\alpha})=\sum_{H} t_{H, p}^{(1)}(\underline{\alpha}) t_{\overline{G / H}, p}^{(2)}(\underline{\alpha}),
$$


где суммирование ведется по всем остовным подграфам $H$ графа $G$, каждая компонента связности которых представляет собой регулярный подграф, и граф $\widehat{G / H}$ не содержит петель,

$$
\begin{aligned}
t_{H, p}^{(1)}(\underline{\alpha})= & \int \prod_{l \in L(G)}\left\|x_{i(l), p}-x_{f(l), p}\right\|_{p}^{\alpha_{l}-2 d} \prod_{v \in V(G)} \chi\left(x_{v, p}\right) d x_{v, p}, \\
t_{H, p}^{(2)}(\underline{\alpha})= & \int \prod_{l \in L(G)}\left\|x_{i(l), p}-x_{f(l), p}\right\|_{p}^{\alpha_{l}-2 d} \times \\
& \times\left(1-\chi_{p}\left(x_{i(l), p}-x_{f(l), p}\right)\right) \prod_{v \in V(G) \backslash\left\{v_{0}\right\}} d x_{v, p},
\end{aligned}
$$

где $v_{0}$ - любая вершина из множества $V(H)$, значение интеграла (10) не зависит от ее выбора. Для интегралов (9), (10) сушествуют явные формулы (см. $[2,11,15])$. Из этих явных формул можно получить следуюшие представления:

$$
t_{H, p}^{(1)}(\underline{\alpha})=\frac{1+Q_{H}^{(1)}\left(p^{-d} ; p^{\operatorname{Ind}\left(H^{\prime}\right)}, H^{\prime} \in \mathcal{H}_{1}(H) \backslash\{G\}\right)}{\prod_{H^{\prime} \in \mathcal{H}_{1}(H)}\left(1-p^{\operatorname{Ind}\left(H^{\prime}\right)}\right)},
$$

где $\mathcal{H}_{1}(H)$ - совокупность нетривиальных регулярных связных подграфов графа $H$, $Q_{H}^{(1)}\left(x ; y_{H^{\prime}}, H^{\prime} \in \mathcal{H}_{1}(H)\right)$ - полином от переменной $x$ и переменных $y_{H^{\prime}}$ с нулевым свободным членом;

$$
\begin{gathered}
t_{H, p}^{(2)}=1, \quad \text { если } \quad V(H)=1, \quad|L(H)|=\varnothing, \\
t_{H, p}^{(2)}(\underline{\alpha})=\frac{Q_{H}^{(2)}\left(p^{-d} ; p^{-\operatorname{Ind}\left(H / H^{\prime}\right)}, H^{\prime} \in \mathcal{H}_{2}(H)\right)}{\prod_{H^{\prime} \in \mathcal{H}_{2}(H)}\left(1-p^{-\operatorname{Ind}\left(H / H^{\prime}\right)}\right)},
\end{gathered}
$$

где $\mathcal{H}_{2}(H)$ - совокупность остовных подграфов графа $H$, каждая компонента связности которых является регулярным подграфом этого графа, $Q_{H}^{(2)}\left(x ; y_{H^{\prime}}, H^{\prime} \in \mathcal{H}_{2}(H)\right)$ - полином с нулевым свободным членом. Заметим, что для любого подграфа $H^{\prime}$ графа $\widehat{G / H}$, каждая компонента связности которого является регулярным подграффом, мы можем написать, что

$$
(\widehat{G / H}) / H^{\prime}=\widehat{G / H^{\prime \prime}},
$$

где $H^{\prime \prime}$ - подграф графа $G$, каждая компонента связности которого есть регулярный подграф. В силу (11) и (12) мы получаем представление

$$
f_{G, p}(\underline{\alpha})=\frac{1+Q_{G}\left(p^{-d} ; p^{\operatorname{Ind}(H)}, H \in \mathcal{H}_{1}(G) ; p^{-\operatorname{Ind}\left(\widehat{G / H^{\prime}}\right)}, H^{\prime} \in \mathcal{H}_{2}^{\prime}(G)\right)}{\prod_{H \in \mathcal{H}_{1}(G)}\left(1-p^{\operatorname{Ind}\left(H^{\prime}\right)}\right) \prod_{H \in \mathcal{H}_{2}^{\prime}(G)}\left(1-p^{-\operatorname{Ind}(\widehat{G / H})}\right)},
$$

где $\mathcal{H}_{2}^{\prime}(G)=\left\{H: H \in \mathcal{H}_{2}(G), \widehat{G / H}\right.$ не содержит петель $\}, Q_{G}\left(x ; y_{H}, H \in \mathcal{H}_{1}(G) ; z_{H^{\prime}}\right.$, $\left.H^{\prime} \in \mathcal{H}_{2}^{\prime}(G)\right)$ - полином с нулевым свободным членом. Из неравенств (6), (7) следует, что бесконечное произведение, задающее $f_{G}(\underline{\alpha})$, сходится.

Докажем теперь, что для любого графа $G$, не содержащего отделимых подграфов, при достаточно большом $d$ сушествует непустая область в пространстве параметров $\underline{\alpha}$, 
для которой выполнены неравенства (6), (7). В работе [16] было доказано, что для любой размерности $d$, в том числе и для $d=1$, сушествует непустая область в пространстве параметров $\underline{\alpha}$, в которой левые части неравенств (6) отрицательны, а левые части неравенств (7) положительны. Сами неравенства (6) и (7) переписываются в виде

$$
\begin{array}{r}
-\operatorname{Re} \sum_{l \in L(H)} \frac{\alpha_{l}}{d}+2|L(H)|-|V(H)|+1<-\frac{1}{d}, \\
-\operatorname{Re} \sum_{l \in L(\widehat{G / H})} \frac{\alpha_{l}}{d}+2|L(\widehat{G / H})|-|V(\widehat{G / H})|+1<-\frac{1}{d} .
\end{array}
$$

Поскольку число подграфов графа $G$ конечно, то отсюда следует утверждение теоремы.

Определим $F_{G}(\varepsilon), \varepsilon \in C$, как $F_{G}(\underline{\alpha}(\varepsilon))$, где $\underline{\alpha}(\varepsilon)=(3 d / 2+\varepsilon, \ldots, 3 d / 2+\varepsilon)$. Такому выбору $\underline{\alpha}$ отвечает адельная фейнмановская амплитуда с пропагатором $|x-y|^{-d / 2+\varepsilon}$. Аналогичные обозначения $f_{G}(\varepsilon), f_{G, p}(\varepsilon)$ будем использовать для интегралов $f_{G}(\underline{\alpha}(\varepsilon))$, $f_{G, p}(\underline{\alpha}(\varepsilon))$.

Теорема 2. Если связный граф $G \varphi^{4}$-теории, имеющий не менее двух внеиних вершин, не содержит собственных двухвосток, то для достаточно больиих значений d существует непустая область значений параметра $\varepsilon$, для которой интеграл $\left(F_{G}(\varepsilon), h\right)$ сходится для любой функции $h$ из пространства Швариа-Брюа.

ДокАЗАТЕЛЬСТво. Легко видеть, что индекс произвольного фейнмановского графа $G^{\prime}$ с пропагатором $|x-y|^{-d / 2+\varepsilon}$ имеет вид

$$
\operatorname{Ind}\left(G^{\prime}\right)=-\left|L\left(G^{\prime}\right)\right| \varepsilon+d\left(1-\left|V\left(G^{\prime}\right)\right|+\frac{n\left(G^{\prime}\right)-\left|E\left(G^{\prime}\right)\right|}{4}\right)
$$

где $n\left(G^{\prime}\right)$ - сумма степеней всех вершин графа $G^{\prime}$ (степенью вершины $v$ мы называем количество внутренних и внешних ребер, инцидентных вершине $v), E\left(G^{\prime}\right)$ - множество внешних ребер графа $G^{\prime}$. Если $H$ - граф̆ $\varphi^{4}$-теории, то формула (13) принимает вид

$$
\operatorname{Ind}(H)=-|L(H)| \varepsilon+d\left(1-\frac{|E(H)|}{4}\right) .
$$

Условие (6) переписывается как

$$
\operatorname{Re} \varepsilon>\frac{1+d(1-|E(H)| / 4)}{|L(H)|}
$$

Если исходный граф не является двухвосткой, то при $\operatorname{Re} \varepsilon>1 / 2$ неравенство (6) имеет место для любого его регулярного подграфа $H$, поскольку $1-E(H) / 4 \leqslant 0$, и либо $|L(H)|>1$, либо $|L(H)|=1$ и $E(H)=6$. Если же исходный граф - двухвостка, то неравенство (6) имеет место при $\operatorname{Re} \varepsilon>\max \{1 / 2,(1+d / 2) /|L(G)|\}$. 
Легко видеть, что

$$
\begin{aligned}
\operatorname{Ind}\left(\widehat{G^{\prime}}\right) & =\operatorname{Ind}\left(G^{\prime}\right)+d\left(\left|V_{\mathrm{ext}}\left(G^{\prime}\right)\right|-1\right)= \\
& =-\left|L\left(G^{\prime}\right)\right| \varepsilon+d\left(\frac{n\left(G^{\prime}\right)-\left|E\left(G^{\prime}\right)\right|}{4}+\left|V_{\mathrm{ext}}\left(G^{\prime}\right)\right|-\left|V\left(G^{\prime}\right)\right|\right) .
\end{aligned}
$$

Если $G^{\prime}=G / H$, граф $G$ удовлетворяет условиям теоремы, а $H$ - его собственный подграфф такой, что каждая компонента связности графа $H$ является регулярным подграфом графа $G$ (эти условия мы будем предполагать выполненными в дальнейшем), то

$$
\frac{n\left(G^{\prime}\right)-\left|E\left(G^{\prime}\right)\right|}{4}+\left|V_{\text {ext }}\left(G^{\prime}\right)\right|-\left|V\left(G^{\prime}\right)\right|=\frac{\left|L\left(G^{\prime}\right)\right|}{2}-\left|V_{\text {int }}\left(G^{\prime}\right)\right|>0,
$$

так как степень каждой внутренней вершины графа $G^{\prime}$ не меньше четырех. Таким образом, для любого графа $G^{\prime}$ можно подобрать такое $d_{1}\left(G, G^{\prime}\right)$, что

$$
\frac{d\left(n\left(G^{\prime}\right)-\left|E\left(G^{\prime}\right)\right| / 4+\left|V_{\text {ext }}\left(G^{\prime}\right)\right|-\left|V\left(G^{\prime}\right)\right|\right)-1}{\left|L\left(G^{\prime}\right)\right|}>\frac{1}{2}
$$

при $d>d_{1}\left(G, G^{\prime}\right)$. Отсюда следует, что если граф $G$ не является двухвосткой, то при $d>\max _{G^{\prime}} d_{1}\left(G, G^{\prime}\right)$ сушествует непустой интервал значений $\varepsilon$, для которого выполнены условия (6) и (7).

Если граф $G$ является двухвосткой, то для фактор-графа $G^{\prime}=G / H$ имеет место альтернатива: либо $\left|V_{\text {ext }}\left(G^{\prime}\right)\right|=2$, и тогда

$$
\frac{n\left(G^{\prime}\right)}{4}-\left|V\left(G^{\prime}\right)\right|+\left|V_{\mathrm{ext}}\left(G^{\prime}\right)\right|-\frac{|E(G)|}{4} \geqslant \frac{3}{2}
$$

либо $\left|V_{\text {ext }}\left(G^{\prime}\right)\right|=1$, и тогда

$$
\frac{n\left(G^{\prime}\right)}{4}-\left|V\left(G^{\prime}\right)\right|+\left|V_{\text {ext }}\left(G^{\prime}\right)\right|-\frac{|E(G)|}{4} \geqslant \frac{1}{2}, \quad\left|L\left(G^{\prime}\right)\right|<|L(G)|
$$

В обоих случаях можно подобрать такое $d_{2}\left(G, G^{\prime}\right)$, что при $d>d_{2}\left(G, G^{\prime}\right)$

$$
\frac{d\left(n\left(G^{\prime}\right)-\left|E\left(G^{\prime}\right)\right| / 4+\left|V_{\text {ext }}\left(G^{\prime}\right)\right|-\left|V\left(G^{\prime}\right)\right|\right)-1}{\left|L\left(G^{\prime}\right)\right|}>\max \left\{\frac{1+d / 2}{|L(G)|}, \frac{1}{2}\right\} .
$$

Теорема доказана. 


\section{3. АНАЛИТИЧЕСКИЕ СВОЙСТВА АДЕЛЬНЫХ АМПЛИТУД $\varphi^{4}$-ТЕОРИИ В НИЗШИХ ПОРЯДКАХ ТЕОРИИ ВОЗМУШЕНИЙ}

В этом разделе мы изучим аналитические свойства некоторых амплитуд $\varphi^{4}$-теории, возникаюших в третьем и четвертом порядках теории возмушений. Действие произвольной фейнмановской амплитуды $F_{G}(\varepsilon)$ на основную функцию $h, h \in S\left(A^{d}\right)$, может быть представлено в виде

$$
\left(F_{G}(\varepsilon), h\right)=m_{G}(\varepsilon) f_{G}(\varepsilon),
$$

где $m_{G}(\varepsilon)$ - мероморфная функция от $\varepsilon$. Область аналитичности амплитуды $F_{G}$ совпадает с областью аналитичности интеграла $f_{G}(\varepsilon)$, аналитические свойства которого мы и будем обсуждать ниже.
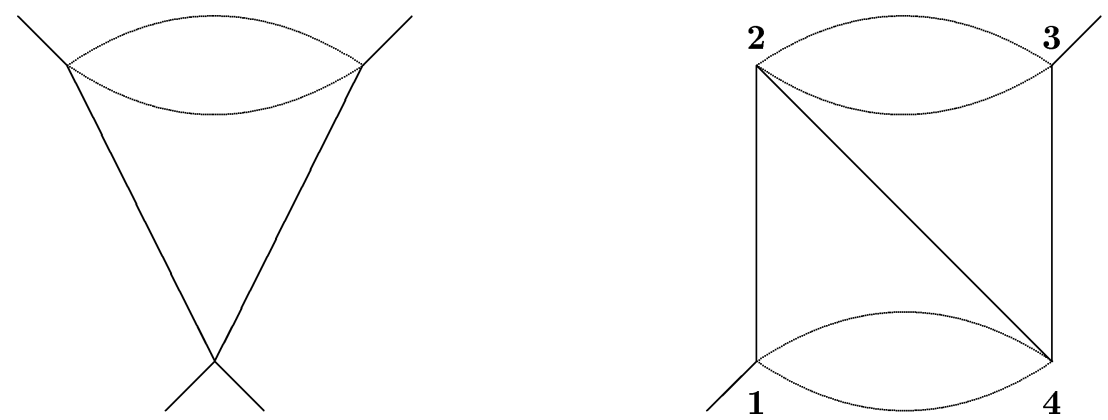

Первый пример фейнмановского интеграла $f_{G}(\varepsilon)$, который не представляется в виде конечного произведения дзета-функций, задается графом $G=G_{1}$, изображенным слева на рисунке. Явные формулы для этого интеграла и вопрос о перенормируемости соответствуюшей фейнмановской амплитуды в точке $\varepsilon=0$ обсуждались в работах $[8,11]$. Явная формула для $f_{G_{1}}(\varepsilon)$ имеет вид

$$
f_{G_{1}}(\varepsilon)=\prod_{p} \frac{\left(1-p^{-d}\right)\left(1-2 p^{-d}+p^{-d / 2-\varepsilon}+p^{-d-2 \varepsilon}-2 p^{-d / 2-3 \varepsilon}+p^{-3 d / 2-3 \varepsilon}\right)}{\left(1-p^{-4 \varepsilon}\right)\left(1-p^{-d / 2-\varepsilon}\right)\left(1-p^{-2 \varepsilon}\right)} .
$$

При $\varepsilon>1 / 2$ и $d \geqslant 2$ интеграл $f_{G_{1}}$ сходится и представляется в виде

$$
\zeta(4 \varepsilon) \zeta\left(\frac{d}{2}+\varepsilon\right) \zeta(2 \varepsilon) \zeta^{-1}(d) \prod_{p=2} T_{G_{1}, p}(\varepsilon)
$$

где

$$
T_{G_{1}, p}(\varepsilon)=1-2 p^{-d}+p^{-d / 2-\varepsilon}+p^{-d-2 \varepsilon}-2 p^{-d / 2-3 \varepsilon}+p^{-3 d / 2-3 \varepsilon} .
$$

Второй пример фейнмановского интеграла, который мы будем рассматривать, задается графом $G_{2}$, изображенным справа на рисунке. Как следует из доказательства теоремы 2, неравенство (6) для граф̆а $G_{2}$ эквивалентно неравенству

$$
\varepsilon>\max \left\{\frac{1}{7}+\frac{d}{14}, \frac{1}{2}\right\}
$$


и несложно показать, что неравенство (7) эквивалентно неравенству

$$
\varepsilon<\frac{3 d / 2-1}{7}
$$

Система неравенств (15), (16) имеет решение при $d>3$. Легко видеть, что

$$
f_{G_{2}, p}(\varepsilon)=f_{G_{2}, p}^{1}(\varepsilon)+f_{G_{2}, p}^{2}(\varepsilon)+2 f_{G_{2}, p}^{3}(\varepsilon)
$$

где

$$
\begin{aligned}
f_{G_{2}, p}^{1}(\varepsilon)= & \int \prod_{l \in L\left(G_{2}\right)}\left\|x_{i(l)}-x_{f(l)}\right\|_{p}^{-d / 2+\varepsilon} \prod_{v \in V\left(G_{2}\right)} \chi_{p}\left(x_{v}\right) d x_{v}, \\
f_{G_{2}, p}^{2}(\varepsilon)= & \int\left\|x_{2}-x_{4}\right\|_{p}^{-d / 2+\varepsilon}\left\|x_{2}\right\|_{p}^{-3(d / 2+\varepsilon)}\left\|x_{4}\right\|_{p}^{-3(d / 2+\varepsilon)}\left(1-\chi_{p}\left(x_{2}\right)\right) d x_{2} \times \\
& \times\left(1-\chi_{p}\left(x_{4}\right)\right) d x_{4}, \\
f_{G_{2}, p}^{3}(\varepsilon)= & \int\left\|x_{1}-x_{2}\right\|_{p}^{-d / 2+\varepsilon}\left\|x_{2}-x_{3}\right\|_{p}^{-d+2 \varepsilon} \prod_{i=1}^{3} \chi_{p}\left(x_{i}\right) d x_{i} \times \\
& \times \int\left\|x_{4}\right\|_{p}^{-4(d / 2+\varepsilon)}\left(1-\chi_{p}\left(x_{4}\right)\right) d x_{4},
\end{aligned}
$$

$x_{i}, i=1, \ldots, 4,-$ переменная, соответствуюшая вершине $i$. Используя технику вычисления $p$-адических фейнмановских интегралов, разработанную в работах $[2,11]$, можно показать, что

$$
\begin{aligned}
f_{G_{2}, p}^{1}(\varepsilon)= & \zeta_{p}\left(-\frac{d}{2}+7 \varepsilon\right)\left\{\left(1-p^{-d}\right)\left(1-2 p^{-d}\right)^{2}+\right. \\
& +\left(1-p^{-d}\right)^{2}\left(1-2 p^{-d}\right)\left[3 \gamma_{p}\left(\frac{d}{2}+\varepsilon\right)+2 \gamma_{p}(2 \varepsilon)+2 \gamma_{p}(4 \varepsilon)\right]+ \\
& +\left(1-p^{-d}\right)^{3}\left[2 \gamma_{p}\left(\frac{d}{2}+3 \varepsilon\right)\left(\gamma_{p}\left(\frac{d}{2}+\varepsilon\right)+\gamma_{p}(2 \varepsilon)+\gamma_{p}(d)\right)+\right. \\
& \left.+2 \gamma_{p}(4 \varepsilon)\left(2 \gamma_{p}\left(\frac{d}{2}+\varepsilon\right)+\gamma_{p}(2 \varepsilon)\right)\right]+ \\
& \left.+\left(1-p^{-d}\right)^{3}\left[\gamma_{p}^{2}(2 \varepsilon)+\gamma_{p}^{2}\left(\frac{d}{2}+\varepsilon\right)+\gamma_{p}\left(\frac{d}{2}+\varepsilon\right) \gamma_{p}(d)\right]\right\}, \\
f_{G_{2}, p}^{2}(\varepsilon)= & \left(1-p^{-d}\right) \gamma_{p}\left(\frac{3 d}{2}-7 \varepsilon\right)\left\{1-2 p^{-d}+\right. \\
& \left.+\left(1-p^{-d}\right) \gamma_{p}\left(\frac{d}{2}+\varepsilon\right)+2\left(1-p^{-d}\right) \gamma_{p}(d-4 \varepsilon)\right\}, \\
f_{G_{2}, p}^{3}(\varepsilon)= & \left(1-p^{-d}\right)^{3} \gamma_{p}(d-4 \varepsilon) \zeta_{p}\left(\frac{d}{2}+\varepsilon\right) \zeta_{p}(2 \varepsilon),
\end{aligned}
$$


где $\gamma_{p}(s)=p^{-s} /\left(1-p^{-s}\right)=-\zeta_{p}(-s)$. Анализируя эти формулы, можно показать, что справедливо представление

$$
\begin{aligned}
f_{G_{2}, p}(\varepsilon)= & T_{G_{2}, p}(\varepsilon) \zeta_{p}\left(-\frac{d}{2}+7 \varepsilon\right) \zeta_{p}(2 \varepsilon) \zeta_{p}(4 \varepsilon) \times \\
& \times \zeta_{p}(d-4 \varepsilon) \zeta_{p}\left(\frac{d}{2}+\varepsilon\right) \zeta_{p}\left(\frac{3}{2 d}-\varepsilon\right) \zeta_{p}\left(\frac{d}{2}+3 \varepsilon\right),
\end{aligned}
$$

где $T_{G_{2}, p}(\varepsilon)=1+p^{-2 \varepsilon}+2 p^{-4 \varepsilon}+Q_{2}(p ; d, \varepsilon), Q_{2}-$ конечная линейная комбинация членов вида $p^{\beta(k, l)}, k \in \mathbb{N}, l \in \mathbb{Z}, \beta(k, l)=-d k / 2+l \varepsilon$, причем в области $0<\operatorname{Re} \varepsilon<3 d / 14-1 / 7$ при $d>3$ для всех $\beta(k, l)$ справедливо неравенство $\operatorname{Re} \beta(k, l)<-1$. Следовательно, при $3 d / 14-1 / 7>\operatorname{Re} \varepsilon>1 / 2, d>3$, интеграл $f_{G_{2}}(\varepsilon)$ сходится и представляется в виде

$$
\begin{aligned}
f_{G_{2}}(\varepsilon)= & \zeta\left(-\frac{d}{2}+7 \varepsilon\right) \zeta(2 \varepsilon) \zeta(4 \varepsilon) \zeta(d-4 \varepsilon) \times \\
& \times \zeta\left(\frac{d}{2}+\varepsilon\right) \zeta\left(\frac{3}{2 d}-\varepsilon\right) \zeta\left(\frac{d}{2}+3 \varepsilon\right) \prod_{p} T_{G_{2}, p}(\varepsilon) .
\end{aligned}
$$

Таким образом, вопрос об аналитичности адельного фейнмановского интеграла $f_{G_{i}}(\varepsilon)$, $i=1,2$, сводится к вопросу об аналитичности бесконечного произведения $\prod_{p} T_{G_{i}, p}(\varepsilon)$.

TеОрема 3. Пусть $P(x)=1+b_{1} x+\cdots+b_{n} x^{n}$ и ряд $\sum_{p}|Q(p ; \varepsilon)|$ сходится при $0<$ $\operatorname{Re} \varepsilon<\varepsilon^{\prime}, \quad \varepsilon^{\prime}>1$. Тогда бесконечное произведение $\prod_{p}\left(P\left(p^{-\varepsilon}\right)+Q(p, \varepsilon)\right)$ сходится при $1<\operatorname{Re} \varepsilon<\varepsilon^{\prime}$ и представимо в виде

$$
\prod_{k=1}^{N} \zeta(k \varepsilon)^{-a_{k}} A_{N}(\varepsilon)
$$

где $a_{k}-$ челье числа, задаваемые формулами

$$
a_{k}=\frac{1}{k} \sum_{l: l \mid k} \mu(l)\left(\sum_{i=1}^{n} \lambda_{i}^{-k / l}\right),
$$

$\lambda_{i}, \quad i=1, \ldots, n-$ корни полинома $P(x), \quad \mu(n)-$ функиия Мёбиуса, а $A_{N}(\varepsilon)$ - аналитическая функиия в области $1 /(N+1)<\operatorname{Re} \varepsilon<\varepsilon^{\prime}$.

ДокАЗАТЕЛЬСтво. Докажем сначала, что полином $P(x)$ может быть представлен в виде

$$
P(x)=(1-x)^{a_{1}}\left(1-x^{2}\right)^{a_{2}} \ldots\left(1-x^{N}\right)^{a_{N}}\left(1+x^{N+1} g(x)\right),
$$

где $a_{k}$ находятся по формулам (17), а $g(x)$ - функция, аналитическая в круге $|x|<1$.

Действительно, для любого ряда $1+b_{m} x^{m}+b_{m+1} x^{m+1}+\cdots$ с целыми коэффициентами $b_{i}$ справедливо представление

$$
1+b_{m} x^{m}+b_{m+1} x^{m+1}+\cdots=(1-x)^{-b_{m}}\left(1+c_{m+1} x^{m+1}+\cdots\right),
$$


где $c_{i}, \quad i=m+1, m+2, \ldots$, также являются целыми коэффициентами. Отсюда следует справедливость разложения вида (18). Вычислим коэффициенты $a_{k}$. Пользуясь представлением

$$
P(x)=\prod_{i=1}^{n}\left(1-\lambda_{i}^{-1} x\right)
$$

прологарифмируем обе части равенства (18). Получаем

$$
-\sum_{k=1}^{\infty} \frac{x^{k}}{k}\left(\sum_{i=1}^{n} \lambda_{i}^{-k}\right)=-\sum_{l=1}^{N} a_{l} \sum_{i_{l}=1}^{\infty} \frac{x^{l i_{l}}}{i_{l}}+\log \left(1+x^{N+1} g(x)\right) .
$$

Приравнивая коэффициенты при степенях $x^{k}, k=1, \ldots, N$, получаем, что

$$
\sum_{i=1}^{n} \lambda_{i}^{-k}=\sum_{l: l \mid k} a_{l} l
$$

Используя формулу обрашения Мёбиуса (см., например, [17]), получаем соотношение (17).

Таким образом, мы можем написать, что

$$
P\left(p^{-\varepsilon}\right)+Q(p, \varepsilon)=\left(1-p^{\varepsilon}\right)^{a_{1}} \ldots\left(1-p^{\varepsilon}\right)^{a_{N}} A_{N}(p, \varepsilon),
$$

где

$$
A_{N}(p, \varepsilon)=1+p^{-(N+1) \varepsilon} g\left(p^{-\varepsilon}\right)+Q(p, \varepsilon)\left(1-p^{-\varepsilon}\right)^{-a_{1}} \ldots\left(1-p^{-N \varepsilon}\right)^{-a_{N}} .
$$

При $\operatorname{Re} \varepsilon>1 /(N+1), p \geqslant 2$

$$
\left|\left(1-p^{-\varepsilon}\right)^{-a_{1}} \ldots\left(1-p^{-N \varepsilon}\right)^{-a_{N}}\right|<C_{1}, \quad\left|g\left(p^{-\varepsilon}\right)\right|<C_{2} .
$$

Отсюда следует сходимость бесконечного произведения

$$
A_{N}(\varepsilon)=\prod_{p} A_{N}(p, \varepsilon)
$$

в полосе $1 /(N+1)<\operatorname{Re} \varepsilon<\varepsilon^{\prime}$. Теорема доказана.

Пусть $\varepsilon=-d / 6+\delta / 3$. Тогда

$$
T_{G_{1}, p}(\varepsilon)=1-2 p^{-\delta}+Q_{1}(p ; d, \delta),
$$

где $Q_{1}(p ; d, \delta)=-2 p^{-d}+p^{-d / 3-\delta / 3}+p^{-2(d+\delta) / 3}+p^{-d-\delta}$. Если $d \geqslant 3$, то из теоремы 3 следует представление

$$
\prod_{p} T_{G_{1}, p}(\varepsilon)=\prod_{k=1}^{N} \zeta(k \delta)^{-a_{k}^{(1)}} A_{N}^{(1)}(\delta)
$$


где

$$
a_{k}^{(1)}=\frac{1}{k} \sum_{l: l \mid k} \mu(l) 2^{k / l}
$$

а функция $A_{N}^{(1)}(\delta)$ аналитична при $\delta>1 /(N+1)$. Заметим, что $a_{k}^{(1)}>0, k=1,2, \ldots$ Поскольку $\zeta(k \delta)$ имеет полюс при $\delta=1 / k$, точка $\varepsilon=-d / 6$ является точкой накопления нулей бесконечного произведения $\prod_{p} T_{G_{1}, p}(\varepsilon)$ (а следовательно, и интеграла $f_{G_{1}}(\varepsilon)$ ). Кажется правдоподобным предположение о том, что вся прямая $\operatorname{Re} \varepsilon=-d / 6$ является границей области аналитичности функции $\prod_{p} T_{G_{1}, p}(\varepsilon)$ (а также всей адельной амплитуды $F_{G_{1}}$ ). Заметим, что физически интересная точка $\varepsilon=0$, отвечающая в евклидовых и $p$-адических теориях бифуркационному значению параметра скейлингового преобразования $\alpha=3 / 2 d$, является полюсом этой адельной амплитуды [8].

Рассмотрим теперь произведение $\prod_{p} T_{G_{2}, p}(\varepsilon)$. Пусть $\varepsilon=\delta / 2$. Если $d>3$, то

$$
\prod_{p} T_{G_{2}, p}(\varepsilon)=\prod_{k=1}^{N} \zeta(k \delta)^{-a_{k}^{(2)}} A_{N}^{(2)}(\delta)
$$

где

$$
a_{k}^{(2)}=\frac{1}{k} \sum_{l: l \mid k} \mu(l)\left(\left(\frac{-1+i \sqrt{7}}{2}\right)^{k / l}+\left(\frac{-1-i \sqrt{7}}{2}\right)^{k / l}\right),
$$

a $A_{N}^{(2)}(\delta)$ аналитична при $1 /(N+1)<\operatorname{Re} \delta<(3 d / 2-1) / 14$. Из (19) следует

$$
\sum_{l: l \mid k} a_{l}^{(2)} l=\left(\frac{-1+i \sqrt{7}}{2}\right)^{k}+\left(\frac{-1-i \sqrt{7}}{2}\right)^{k}
$$

Заметим, что $(-1+i \sqrt{7}) / 2=2 e^{i \varphi}$, где $0<\varphi<\pi$. Так как в последовательности $\lambda_{1}^{-k}+\lambda_{2}^{-k}=2^{k / 2+1} \cos (k \varphi), k=1,2, \ldots$, имеется бесконечное число сколь угодно больших по модулю положительных и отрицательных членов, то в последовательности $a_{k}^{(2)}$ также имеется бесконечное число как положительных, так и отрицательных членов. Отсюда, в частности, следует, что точка $\varepsilon=0$ является точкой накопления нулей функции $f_{G_{2}}(\varepsilon)$, расположенных на луче $\operatorname{Im} \varepsilon=0, \operatorname{Re} \varepsilon>0$.

Как известно из теории дзета-функции Римана $[17$, гл. IX], для любой сколь угодно малой окрестности любой точки на мнимой оси можно подобрать такое $k_{0}$, что функция $\zeta(k \delta)$ имеет нуль в этой окрестности при $k>k_{0}$. Если справедлива гипотеза Римана о нулях дзета-функции, то вешественные части нулей функции $\zeta(k \delta)$ равны $1 /(2 k)$, следовательно, все эти нули различны. Таким образом, в силу бесконечности числа отрицательных членов последовательности $a_{k}^{(2)}$, при условии справедливости гипотезы Римана, в окрестности любой точки на мнимой оси имеется бесконечно много нулей произведения $\prod_{p} T_{G_{2}, p}(\varepsilon)$. В этом случае прямая $\operatorname{Re} \varepsilon=0$ является естественной границей области аналитичности функции $f_{G_{2}}(\varepsilon)$ и всей адельной амплитуды $F_{G_{2}}$.

Приведенные примеры показывают, что в отличие от обычных (евклидовых) и $p$-адических фейнмановских амплитуд адельные амплитуды могут не иметь аналитического 
продолжения по степени пропагатора во всю комплексную плоскость. Более того, интересные с физической точки зрения значения степени пропагатора (например, $\varepsilon=0$ ) могут лежать на гранище области аналитичности. Возникает нетривиальный вопрос о том, как понимать процедуру перенормировки таких амплитуд.

Благодарности. Работа выполнена при финансовой поддержке Российского фонда фундаментальных исследований (гранты № 97-01-00375 и № 99-01-00467).

\section{Список литературы}

[1] И. В. Волович. ТМФ. 1987. Т. 71. С. 337-340.

[2] E. Yu. Lerner, M. D. Missarov. Commun. Math. Phys. 1989. V. 121. P. 35-48.

[3] В.С. Владимиров, И. В. Волович, Е. И. Зеленов. р-Адический анализ и математическая физика. М.: Наука, 1994.

[4] L. Brekke, P. G. O. Freund. Phys. Rep. 1993. V. 233. № 1. P. 2-66.

[5] Yu. I. Manin. Reflections on Arithmetical Physics. In: Conformal Invariance and String Theory. Eds. P. Dita, V. Gergescu. Boston: Academic Press, 1989. P. 293-303.

[6] P. G. O. Freund, E. Witten. Phys. Lett. B. 1987. V. 199. P. 191-194

[7] В. С. Владимиров. УМН. 1993. Т. 48. № 6. С. 3-38.

[8] M. D. Missarov. Phys. Lett. B. 1991. V. 272. P. 36-38.

[9] И. М. Гельфанд, М.И. Граев, И. И. Пятецкий-Шапиро. Теория представлений и автоморффые функции. Обобщенные функции. Вып. 6. М.: Наука, 1966.

[10] M. D. Missarov. Ann. Inst. H. Poincaré. 1989. V. 49. P. 357-367.

[11] M.D. Missarov. Renormalization group and renormalization theory in $p$-adic and adelic scalar models. In: Dynamical Systems and Statistical Mechanics. Adv. Sov. Math. V.3. Ed. Ya. G. Sinai. Providence, RI: Amer. Math. Soc., 1991. P. 143-161.

[12] О. И. Завьялов. Перенормированные диаграммы Фейнмана. М.: Наука, 1979.

[13] В. А. Смирнов. Перенормировка и асимптотические разложения фейнмановских амплитуд. М.: Изд-во МГУ, 1990.

[14] Э. Ю. Лернер. ТМФ. 1995. Т. 102. № 3. С. 367-377.

[15] Э. Ю. Лернер. ТМФ. 1995. Т. 104. № 3. С. 371-392.

[16] E. R. Speer. Ann. Inst. H. Poincaré. 1973. V. 23. P. 1-21.

[17] E. К. Титчмари. Теория дзета-функции Римана. М.: ИЛ, 1953.

Поступила в редакцию 6.XII.1999 г. 